\title{
Multi-band optical variability studies of Blazars*
}

\author{
Aditi Agarwal ${ }^{\dagger}$
}

Indian Institute of Astrophysics, Bangalore - 560034, India

\begin{abstract}
To search for optical variability on a wide range of timescales, we have carried out photometric monitoring of a dozen blazars. CCD magnitudes in B, V, R and I pass-bands were determined for $>10,000$ new optical observations from 300 nights made during $2011-2016$, with an average length of $\sim 4 \mathrm{~h}$ each, using seven optical telescopes: four in Bulgaria, one in Greece, and two in India. We measured multiband optical flux and colour variations on diverse timescales. Blazar variability studies helped us in understanding their nature and extreme conditions within the emission region. To explain possible physical causes of the observed spectral variability, we also investigated spectral energy distributions using B, V, R, I, J and K pass-band data.
\end{abstract}

\section{Introduction}

Active galactic nuclei (AGNs) are generally characterized by extremely high luminosities as they are powered by accretion of matter onto a super massive black hole (SMBH) surrounded by an accretion disk (AD) which yields high radiative efficiencies. Powerful emission from these active galaxies spans the entire electromagnetic (EM) spectra, from radio to very high energy $\gamma$-rays, thus distinguishing them from all other regular galaxies whose radiation is pre-dominantly in infra red from dust, optical due to stars or UV owing to nebulae and stars.

Blazars constitute a subgroup of radio-loud Active Galactic Nuclei that are distinguished by outstanding properties such as a dominant nonthermal continuum and violent variability over the entire EM spectrum, often accompanied by high degrees of polarization. Blazars are classified as flat spectrum radio quasars (FSRQs) if they have prominent emission lines in their spectra and as BL Lacertae objects (BL Lacs) if they have largely featureless optical spectra (Blandford \& Ress 1978). Blazars are understood to have their relativistic jet axis aligned at small angles $\left(\leq 10^{\circ}\right)$ to the observer's line of sight (LOS) (e.g., Urry \& Padovani 1995) which produces relativistic beaming. Blazars have been found to be variable on all accessible timescales ranging from few minutes through days and months to decades. The pronounced variability throughout the Electromagnetic spectrum, has been divided into three classes: flux changes over a time scale of few tens of minutes to less than a day are called intra-day variability (IDV; Wagner \& Witzel 1995, Agarwal et al. 2015); those from several days to few weeks are known as short time variability (STV; Lainela et al. 1999), while long term variability (LTV) covers flux changes from several months to many years (e.g., Gupta et al. 2004, Agarwal \& Gupta 2015).

${ }^{*}$ Blazars in optical regime

†aditiagarwal.phy@gmail.com 


\section{Observations and Data Reduction}

For this study, observations of the blazars covering optical B, V, R, and I passbands were performed using seven telescopes, two in India, one in Greece, and four in Bulgaria. For image pre-processing, photometric observations obtained with the above telescopes were bias subtracted and twilight flat fielded followed by cosmic ray removal.

We performed the preceding steps using $\mathrm{IRAF}^{1}$ software. The next stage is to extract the target's magnitude from the rectified CCD intensity array using the Dominion Astronomical Observatory Photometry (DAOPHOT II) software. Aperture photometry was performed using DAOPHOT II for different aperture radii, i.e., $\sim 1 \times$ FWHM, $2 \times$ FWHM, $3 \times$ FWHM, and $4 \times$ FWHM, out of which aperture $2 \times$ FWHM was selected to get instrumental magnitude of the source, giving the best $\mathrm{S} / \mathrm{N}$ ratios.

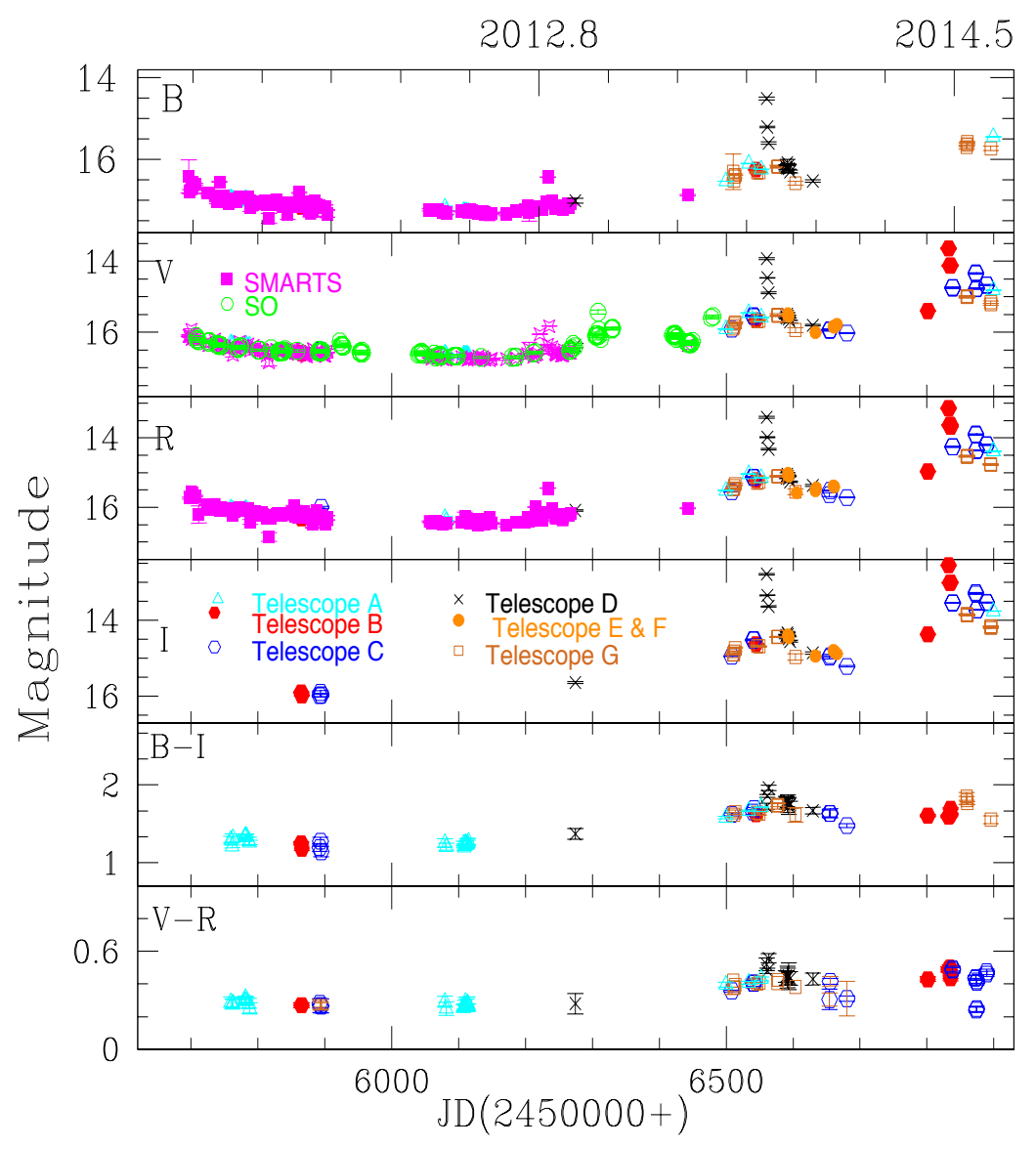

Figure 1: Short/Long-term flux and colour variability LCs of 3C 454.3 in the optical regime. Different colours denote data from different observatories: Cyan $=1.3-\mathrm{m}$ (Skinakas, Greece), Red $=2-\mathrm{m}$ (Rozhen, Bulgaria), Blue $=50 / 70-\mathrm{cm}(\mathrm{NAO}$, Bulgaria $)$, Black $=60-\mathrm{cm}$ (Belogradchik, Bulgaria), Dark orange $=1.3-\mathrm{m}($ ARIES, India) $\& 1.04-\mathrm{m}$ (ARIES, India), Chocolate $=60-\mathrm{cm}(\mathrm{NAO}$, Bulgaria $)$, Magenta $=$ SMARTS, Yellow $=$ Arizona.

\footnotetext{
${ }^{1}$ IRAF is distributed by the National Optical Astronomy Observatories, which are operated by the Association of Universities for Research in Astronomy, Inc., under cooperative agreement with the National Science Foundation.
} 


\section{Analysis Techniques}

We have studied the variability properties of several blazars in different optical bands using a series of mathematical tests namely C-Test, F test and $\chi^{2}$ test (e.g., Agarwal et al. 2015, Agarwal et al. 2016). The modest number of measurements we could make in most bands made use of the ANOVA technique (de Deigo 2010) in-feasible. In order to claim the presence or absence of variability during the observations, we have selected 0.99 and 0.999 confidence levels.

To characterize the variability of the source in all light curves (LCs), we calculated the variability amplitude parameter $A$. This was introduced by Heidt \& Wagner (1996), and is defined as

$$
A=100 \times \sqrt{\left(A_{\max }-A_{\min }\right)^{2}-2 \sigma^{2}}(\%),
$$

where $A_{\max }$ and $A_{\min }$ are the maximum and minimum values in the differential LCs of the blazar, and $\sigma$ is the average measurement error.

\section{Flux and colour variability Results}

Variability at all timescales is one of the distinctive properties of AGNs and variability studies have been very useful in understanding their nature during the extreme conditions within the emission region. On intraday timescales, we found the sources to be variable during most of the nights, which helped us to probe the location and physical processes related to the emission taking place in the targets. The STV was relatively weak during our whole monitoring period. We also examined the colour variability in blazars on minutes to yearly timescales. Strong intra-night colour variations were found for some nights, but no consistent colour variations were found on a yearly basis. Fig. 1 displays STV/LTV flux and colour variability LCs of 3C 454.3 in optical passbands.

As optical flux variations in blazars are often followed by spectral changes (e.g. Marscher \& Gear 1985), studying the relationship between colour index and the source magnitude is a useful tool to understand the origin of variability in blazars allowing us to ascribe these variations to either disk or jet based physical scenarios. For this we investigated the colour - magnitude relationship for all blazars from intraday to few months timescales. Bluer-when-brighter (BWB) trend was dominant during our observations. One such example is displayed in Fig. 2 for the blazar 3C 454.3 where $\mathrm{V}$ band magnitude is given on the $\mathrm{x}$-axis while different colour indices on the $\mathrm{y}$-axis. Presence of colour trends (either redder-when-brighter or bluer when-brighter) in blazars can be explained due to superposition of both blue and red emission components where the red contribution can be attributed to the synchrotron radiation from the jet while the blue one could come from the thermal emission from the AD around the central region. When Doppler boosted jet synchrotron emission dominates over the $\mathrm{AD}$ emission, the bluer-when brighter trend can be explained by acceleration of relativistic particles or due to injection of fresh non thermal electrons with a harder energy distribution.

\subsection{Spectral Energy Distributions (SEDs)}

Blazars broadband SEDs consist of two well-defined components extending from radio upto gammarays and sometimes to $\mathrm{TeV}$ frequencies. The low energy spectrum (radio to UV/X ray) is generally supposed to arise due to synchrotron emission from the relativistic electrons of the jet while the inverse Compton scattering of the synchrotron emission and/or external photons is responsible for high energy peak (extending to $\gamma$-rays)(Coppi \& Aharonian 1999). Differences in the physical parameters of the relativistic jet causes spectral changes in the emitting electrons which is the reason for SED changes thus rendering SED studies very useful. 


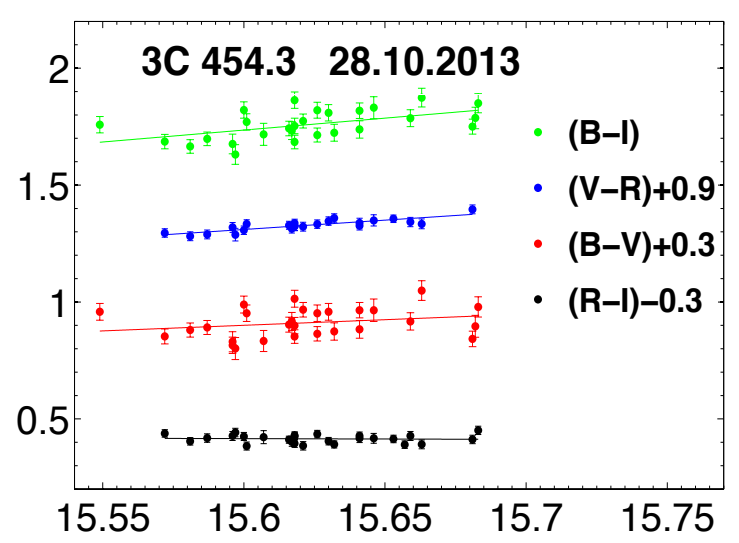

Figure 2: Color-indices (y-axis) against $\mathrm{V}$ band magnitude (x-axis) on intraday timescales for 3 C 454.3 .

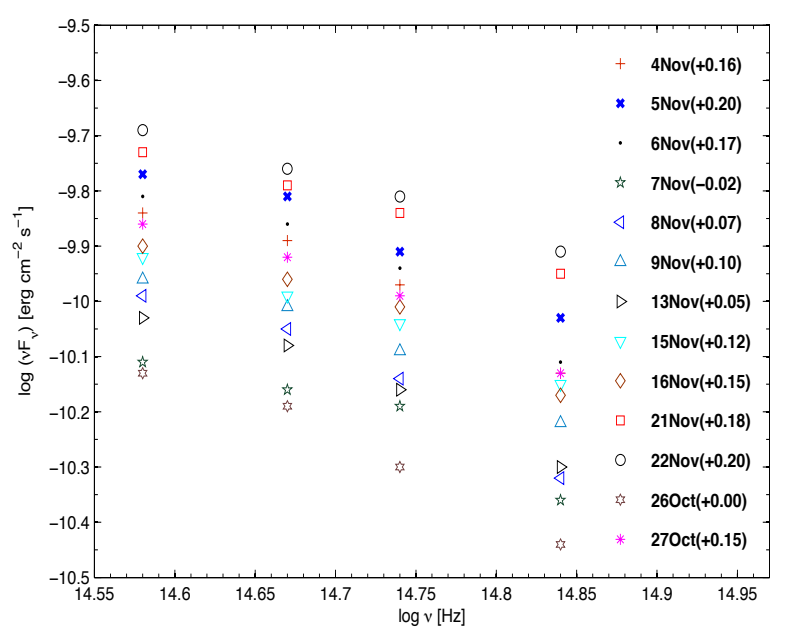

Figure 3: SED results for BL Lacertae at optical frequencies. Different symbols are used for each epoch mentioned in the figure along with the offset used.

To look for signs of multiple components contributing to synchrotron emission in the optical part of the SED or study anomalous spectral shapes, NIR - optical spectral properties of each blazar were studied by generating and analysing SEDs for each night with sufficient data points in the B, V, R, $\mathrm{I}, \mathrm{J}$ and $\mathrm{K}$ filters. We de-reddened the calibrated magnitudes of the targets by subtracting Galactic absorption using the NED extinction calculator (Bessell et al., 1998).

Fig. 3 shows optical SEDs for the source BL Lacertae, built with our B, V, R, I data sets for different epochs with different brightness levels. Significant variations were seen in the SEDs during the entire observation period. Since the optical band for this source lies near the peak of the synchrotron component of the SED, the optical variability reflects acceleration processes acting on the highest energy electrons.

Although with the advent of large telescopes many aspects of these sources are now clear, nevertheless, many still remain uncertain like the underlying radiation processes, their formation, evolution, and taxonomy which further requires systematic studies. Through this research, we aim to gain insight on various aspects of blazar, including the above mentioned ones.

\section{References}

Agarwal A., Gupta A. C. 2015, MNRAS, 450, 541

Agarwal A., Gupta A. C., Bachev R. et al. 2015, MNRAS, 451, 3882

Agarwal A., Gupta A. C., Bachev R. et al. 2016, MNRAS, 455, 680

Bessell M. S., Castelli F., Plez B. 1998, A\&A, 333, 231

Blandford R. D., Rees M. J. 1978, PhyS, 17, 265

Coppi P. S., Aharonian F. A. 1999, ApJ, 521, L33

de Diego J. A. 2010, AJ, 139, 1269

Gupta A. C., Banerjee D. P. K., Ashok N. M., Joshi U. C. 2004, A\&A, 422, 505

Heidt J., Wagner S. J. 1996, A\&A, 305, 42

Lainela M., Takkalo L. O., Sillanpää A. et al. 1999, ApJ, 521, 561

Marscher A. P., Gear W. K. 1985, ApJ, 298, 114

Urry C. M., Padovani P. 1995, PASP, 107, 803

Wagner S. J., Witzel A. 1995, ARA\&A, 33, 163 